\title{
A CHARACTERIZATION OF OSCILLATORY PROCESSES AND THEIR PREDICTION
}

\section{MANDREKAR ${ }^{1}$}

ABstract. The oscillatory stochastic processes recently studied by Priestley are characterized as deformed stationary curves in a Hilbert space. This characterization leads to the simple time domain proof of prediction and moving average representation for these stochastic processes in terms of the associated stationary curve.

In [6], A. N. Kolmogorov studied second order stationary processes as curves in Hilbert space. The idea again occurred in Cramér [2] for nonstationary processes. In this note we characterize the oscillatory processes introduced by Priestley [8] as deformed stationary curves in a Hilbert space and give a simple geometric solution for the prediction problem for such curves. As a consequence of this one can easily derive the analytic results on prediction in [1], thus providing a simple and more general solution for the prediction problem of oscillatory processes. Also our characterization makes the definition of the time dependent spectral distribution an obvious generalization of the stationary case. For the sake of being specific we consider throughout the continuous parameter case.

1. Definition. Let $H$ be a Hilbert-space and $R$ be the space of real numbers with the usual topology. We say that

(a) $x$ is a continuous curve in $H$, if $x$ is a continuous map of $R$ into $H$.

(b) $y$ is a stationary continuous curve in $H$ if (i) $y$ is a continuous curve in $H$, (ii) $(y(t), y(s))$ is a function of $t-s$.

With every curve $x$ in $H$ we associate the following subspaces of $H$ :

$$
\begin{aligned}
H_{x}(t) & =\Im\{x(\tau), \tau \leqq t\},{ }^{2} \\
H_{x}(+\infty) & =\Im\{x(\tau), \tau \in R\}, \\
H_{x}(-\infty) & =\bigcap_{t} H_{x}(t) .
\end{aligned}
$$

Received by the editors April 26, 1971.

AMS 1970 subject classifications. Primary 60G25; Secondary 47A50.

Key words and phrases. Oscillatory stochastic processes, stationary curves in Hilbert space, prediction, moving average representation.

${ }^{1}$ Research supported by NSF Grant GP-11626.

$2 \mathfrak{S}\{\}$ denotes the subspace generated by vectors \{\} .

(c) American Mathematical Society 1972 
If the curve is continuous $H_{x}(+\infty)$ is separable. We say that a curve $x$ in $H$ is purely nondeterministic if $H_{x}(-\infty)=\{0\}$ and is said to be a deformed stationary curve if there exists a stationary continuous curve $y$ in $H$ and for each $t \in R, A(t)$ on $H_{y}(+\infty)$ into $H_{y}(+\infty)$ such that

$$
\begin{aligned}
& \text { (a) } \mathscr{D}(A(t)) \supset\{y(t),-\infty<t<+\infty\}, \\
& \text { (b) } x(t)=A(t) y(t), \quad t \in R .
\end{aligned}
$$

If $H=L_{2}(\Omega, P)$ the space of square-integrable functions with respect to $P$, the oscillatory processes are defined in [8] (see Note A below) as

$$
x_{t}=\int_{-\infty}^{+\infty} e^{i t u} a_{t}(u) Z(d u)
$$

where (i) $Z(\cdot)$ is an orthogonal countably additive (c.a.) set function with values in $H$, (ii) the integral in (3) is the stochastic integral (Doob [3, p. 208]), (iii) for each $t \in R, \int_{-\infty}^{+\infty}\left|a_{t}(u)\right|^{2} \rho(d u)<\infty$ where

$$
\rho(A)=(Z(A), Z(A))_{L_{2}(\Omega)}
$$

$A \in B(R)$, Borel subsets of $R$.

Let us now define

$$
y(t)=\int_{-\infty}^{+\infty} e^{i t u} Z(d u)
$$

Then clearly $[3$, p. 526] $y$ is a stationary continuous curve in $H$. Using Stone's Theorem $[9, \mathrm{p} .383]$ and uniqueness of the Fourier representation one obtains $Z(A)=E(A) y_{0}$ for $A \in B(R)$, where $E$ is the spectral measure associated with the shift group defined for each $s \in R$ by

$$
U_{t} y(s)=y(t+s), \quad t \in R,
$$

on $H_{y}(+\infty)$ into $H_{y}(+\infty)$. Furthermore $H_{y}(+\infty)=\Im\left\{U_{t} y_{0}, t \in R\right\}$.

The following is our characterization of oscillatory processes.

6. THEOREM. $x$ is an oscillatory curve in $H$ iff $x$ is a deformed stationary curve such that for each $t, A(t) U_{s} h=U_{s} A(t) h$, for $h \in \mathscr{D}(A(t))$ and for all $s \in R$.

Proof. Let $x_{t}$ be oscillatory. Then $y(t)$ defined in (4) is a continuous stationary curve. Furthermore $y_{t}=\int_{-\infty}^{+\infty} e^{i t u} E(d u) y_{0}$ where $E(\cdot)$ is the spectral measure associated with a continuous unitary group

$$
\left\{U_{t},-\infty<t<+\infty\right\}
$$

given in (5) ([9, p. 383]). Define, for each $t \in R$,

$$
A(t)=\int_{-\infty}^{+\infty} a_{t}(u) E(d u) .
$$


Then by [4, p. 1196], $A(t)$ is closed. For each $t, \mathscr{D}(A(t)) \supset\{y(s), s \in R\}$. By the definition of the spectral integral and the fact $U_{s} E(A)=E(A) U_{s}$ for $A \in B(R)$, we obtain that, for each $t \in R, A(t) U_{s} h=U_{s} A(t) h$ for $h \in \mathscr{D}(A(t))$ and for all $s \in R$. Furthermore,

$$
\begin{aligned}
A(t) y(t) & =\int_{-\infty}^{+\infty} a_{t}(u) E(d u)\left[\int_{-\infty}^{+\infty} e^{i t u} E(d u) y_{0}\right] \\
& =\int_{-\infty}^{+\infty} a_{t}(u) e^{i t u} E(d u) y_{0}=x_{t}
\end{aligned}
$$

since $E(A) \int_{-\infty}^{+\infty} e^{i t u} E(d u) y_{0}=\int_{A} e^{i t u} E(d u) y_{0}, A \in B(R)$.

To prove the converse, we observe that since $A(t) U_{s} h=U_{s} A(t) h$ for $h \in \mathscr{D}(A(t)) \supseteq\{y(t),-\infty<t<+\infty\}$ and $H_{y}(+\infty)=\subseteq\left\{U_{s} y_{0}, s \in R\right\}$ we obtain by $\left[7\right.$, p. 549] that there exists Borel measurable function $a_{t}(\cdot)$ such that

$$
A(t)=\int_{-\infty}^{+\infty} a_{t}(u) E(d u)
$$

The fact that $\mathscr{D}(A(t)) \supseteq\{y(t),-\infty<t<+\infty\}$ [4, p. 1196] implies, for each $t \in R, \int_{-\infty}^{+\infty}\left|a_{t}(u)\right|^{2}\left\|E(d u) y_{0}\right\|^{2}<\infty$. Hence we obtain

$$
\begin{aligned}
x_{t} & =\int_{-\infty}^{+\infty} a_{t}(u) E(d u) \int_{-\infty}^{\infty} e^{i t u} E(d u) y_{0} \\
& =\int_{-\infty}^{+\infty} e^{i t u} a_{t}(u) E(d u) y_{0}
\end{aligned}
$$

giving $x_{t}$ oscillatory.

REMARK. We now observe that since $x_{t}=U_{t} A(t) y_{0}$ the spectrum of $x_{t}$ is given by $\left\|E(A) A(t) y_{0}\right\|^{2}$. This definition of spectrum was given in [8].

We now give sufficient conditions for a deformed stationary curve $x=A(\cdot) y$ to be purely nondeterministic.

8. Corollary. Let $x$ be a deformed stationary curve of the form (2). If $y$ is purely nondeterministic and, for each $t, A(t) H_{y}(t) \subseteq H_{y}(t)$, then $x$ is purely nondeterministic.

9. Corollary. Let $x$ be a deformed stationary curve of the form (2). Assume that for each $t \in R$, (i) $A(t) H_{y}(t) \subseteq H_{y}(t)$; (ii) $\left.A(t)\right|_{H_{y}(t)}$ is invertible; (iii) $A_{t}^{-1} H_{y}(t) \subseteq H_{y}(t)$, then $x$ is purely nondeterministic iff $y$ is purely nondeterministic.

10. Corollary. Under the same assumptions as in Corollary 9, $x(t)$ has multiplicity one in the sense of Cramér [2]. 
11. Corollary (Moving average representation). Let $x$ be $a$ deformed stationary curve with associated stationary curve purely nondeterministic. Assume that $A(t)$ satisfies the assumptions of Corollary 8. Then

(a) $y_{t}=\int_{-\infty}^{t} f(t-u) \xi(d u)$ where $\xi(\cdot)$ is a c.a. set function with values in $H_{y}(+\infty)$ such that $H_{y}(t)=\Im\{\xi(A), A \in B(R), A \subset(-\infty, t]\} ;$ for $A, A^{\prime} \in B(R)$, $\left(\xi(A), \xi\left(A^{\prime}\right)\right)=c \mu\left(A \cap A^{\prime}\right)$ with $\mu$ being the Lebesgue measure and

$$
\int_{-\infty}^{0}|f(u)|^{2} \mu(d u)<\infty
$$

(b) $x_{t}=\int_{-\infty}^{t} h(t, u) \xi(d u)$ where $\xi$ is as in (a).

Proof. (a) is due to Karhunen [5].

(b) From the fact, $x(t) \in \subseteq\{\xi(A), A \in B(R), A \subset(-\infty, t]\}$ we get

$$
x(t)=\int_{-\infty}^{t} h(t, u) \xi(d u) .
$$

Corollaries 8 and 11 give generalizations of the main results of [1] to the deformed stationary curves. The identification of the representation in Corollary 11(b) under additional conditions of [1, pp. 572-573] is trivial and hence omitted.

REMARK. The discrete parameter case can be handled in a parallel way. All one needs is again the Stone-von Neumann theorem on commutants [7].

Note A. We note here that our definition of oscillatory processes differs from that of [8]. The class of oscillatory processes defined in [8] included an extra condition that the modulus of the Fourier transform of $a_{t}(u)$ must have an absolute maximum of zero frequency. This problem is connected with the uniqueness of the representation in Theorem 6 and is connected with the spectral analysis of the process as a time series. It has however no bearing on the prediction problem studied here and hence our results here do include the results in [1].

ACKNOWLEDGEMENT. I would like to thank the referee for comments which led to the addition of Note A which clarifies the relation of this paper to [8].

\section{REFERENCES}

1. N. A. Abdrabbo and M. B. Priestley, On the prediction of non-stationary processes, J. Roy. Statist. Soc. Ser. B 29 (1967), 570-585. MR 36 \#4758.

2. H. Cramér, Stochastic processes as curves in Hilbert space, Teor. Verojatnost. i Primenen. 9 (1964), 193-204. MR 30 \#613.

3. J. L. Doob, Stochastic processes, Wiley, New York, 1953. MR 15, 445.

4. N. Dunford and J. T. Schwartz, Linear operators. II: Spectral theory. Self adjoint operators in Hilbert space, Interscience, New York, 1963. MR 32 \#6181. 
5. K. Karhunen, Über lineare Methoden in der Wahrscheinlichkeitsrechnung, Ann. Acad. Sci. Fenn. Ser. A.I. Math.-Phys. No. 37 (1947), 79 pp. MR 9, 292.

6. A. N. Kolmogorov, Stationary sequences in Hilbert's space, Bull. Moskov. Gos. Univ. Mat. 2 (1941), no. 6, 40 pp. (Russian) MR 5, 101; 13, 1138.

7. P. Masani, The normality of time-invariant, subordinative operators in a Hilbert space, Bull. Amer. Math. Soc. 71 (1965), 546-550. MR 31 \#1567.

8. M. B. Priestley, Evolutionary spectra and non-stationary processes, J. Roy. Statist. Soc. Ser. B 27 (1965), 204-237. MR 33 \#8026.

9. F. Riesz and B. Sz.-Nagy, Leçons d'analyse fonctionnelle, Akad. Kiadó, Budapest, 1953; English transl., Ungar, New York, 1955. MR 14, 286; MR 17, 175.

Department of Statistics and Probability, Michigan State University, East LANSING, MichigAN 48823 